\title{
Q-OPERATORS FOR HIGHER SPIN EIGHT VERTEX MODELS WITH AN EVEN NUMBER OF SITES
}

\author{
TAKASHI TAKEBE \\ Dedicated to Professor Evgeny Sklyanin \\ on the occasion of his sixtieth birthday.
}

\begin{abstract}
We construct the $Q$-operator for generalised eight vertex models associated to higher spin representations of the Sklyanin algebra, following Baxter's 1973 paper. As an application, we prove the sum rule for the Bethe roots.
\end{abstract}

\section{INTRODUCTION}

The $Q$-operator was introduced by Baxter [1] in 1972 as an auxiliary tool to find eigenvalues of the transfer matrix of the eight vertex model. It satisfies the $T Q$-relation,

$$
T(u) Q(u)=Q(u) T(u)=h_{-}(u) Q(u-2 \eta)+h_{+}(u) Q(u+2 \eta),
$$

with the transfer matrix $T(u)$ and commutes with itself: $\left[Q(u), Q\left(u^{\prime}\right)\right]=$ 0 . (The functions $h_{ \pm}(u)$ are defined in (2.8).) Under the assumption of semisimplicity of $T(u)$ and $Q(u)$ the $T Q$-relation leads to equations for the zeros of $Q(u)$, which eventually give the eigenvalues of $T(u)$. In 1973 Baxter constructed another $Q$-operator for the eight vertex model with an even number of sites in [2] and explained the relation with the Bethe Ansatz method in [4]. Since then a huge number of works have been devoted to construction and analysis of $Q$-operators of various models.

The goal of the present paper is to generalise Baxter's construction of the $Q$-operator in [2] to the higher spin case.

In [27] a generalisation of the eight vertex model was proposed by means of higher spin representations of the Sklyanin algebra introduced by Sklyanin in [26]. (The eight vertex model is the spin $1 / 2$ case.) It was shown that the algebraic Bethe Ansatz by Takhtajan and Faddeev 31, which is an elegant reformulation of Baxter's construction of the eigenvectors in [2], 3], [4], can be applied to the higher spin case as well.

2010 Mathematics Subject Classification. 82B23; 81R50.

Key words and phrases. $Q$-operator, higher spin eight vertex model, Sklyanin algebra. 
This model was further studied in [28] and [29] by the algebraic Bethe Ansatz but a certain property of Bethe roots was left unproved. Baxter showed in [1] that the sum of Bethe roots satisfies integrality condition, using holomorphicity of the $Q$-operator. A similar property for the higher spin generalisation was conjectured in [28] and proved under several ad hoc assumptions. One of the motivations of the construction of the $Q$-operator for the generalised models is to prove this sum rule.

Remarkably, although Baxter's construction of the $Q$-operator ([2], $\S 10.5$ of [5]) seems to depend heavily on the matrix structure of the transfer matrix of the eight vertex model, it turns out that we can apply his method to our model mutatis mutandis. In fact,

- the auxiliary matrix $M_{\lambda}(v)$ (3.1) which makes an off-diagonal block of the $L$-matrix degenerate is the same for any spin and thus the same as that for the eight vertex model;

- the null vector of the degenerate off-diagonal block of the twisted $L$-matrix is the local pseudo vacuum vector (or the intertwining vector) $\omega_{\lambda}(u ; v)$ (3.4) used in the algebraic Bethe Ansatz [27], [29], as is the case with the eight vertex model;

- the Hermitian conjugate with respect to the Sklyanin form (A.10) introduced in [26] plays the role of the transpose of matrices in Baxter's construction;

- the commutation relation (3.27) of $Q_{R}$ and $Q_{L}$, intermediate objects in the construction of the $Q$-operator, is proved by using Baxter's argument with the help of Rosengren's theories on the elliptic $6 j$-symbols [24] and the Sklyanin form (the Sklyanin invariant integration) [23].

This paper is organised as follows: in 92 we recall the model introduced in [27 by defining its transfer matrix. The construction of the $Q$-operator in $\$ 3$ is divided into four steps as in [5]. As a first step, in 3.1 we construct an operator $Q_{R}(u)$ satisfying the half of the $T Q$ relation by using the local pseudo vacuum vectors. The other half of the $T Q$-relation (or the " $Q T$ "-relation) is satisfied by another operator $Q_{L}(u)$, which is defined by the Hermitian conjugate of $Q_{R}(u)$ in $₫ 3.2$. The most complicated part is the next step in $\$ 3.3$, the proof of the commutation relation (3.27) of $Q_{R}(u)$ and $Q_{L}(u)$. The main lemma for this proof is proved in Appendix $\mathrm{B}$. Once the commutation relation is proved, the rest of the construction in 33.4 is a routine work. As an application of the $Q$-operator, we rederive the Bethe Ansatz equation in 27] and prove the sum rule of the Bethe roots in \&4. We make several concluding remarks with comments on related works in the final section $\$ 5$. All the necessary facts about the Sklyanin algebra are collected in Appendix A.

Notations. Throughout this paper we use the following notations and symbols. 
- $N \in 2 \mathbb{Z}_{>0}$ : the number of sites. We consider only even $N$.

- $l \in \frac{1}{2} \mathbb{Z}_{>0}$ : the spin of the representation at each site.

- $\tau \in i \mathbb{R}_{>0}$; the elliptic modulus, which is purely imaginary.

- $\eta \in[-1 / 2(2 l+1), 1 / 2(2 l+1)]$ : anisotropy parameter.

- The notations for the theta functions are the same as those in Sklyanin's papers [25], [26] (cf. [20]):

$$
\theta_{a b}(z, \tau)=\sum_{n \in \mathbb{Z}} \exp \left(\pi i\left(\frac{a}{2}+n\right)^{2} \tau+2 \pi i\left(\frac{a}{2}+n\right)\left(\frac{b}{2}+z\right)\right) .
$$

(cf. Jacobi's notation (e.g., [32]): $\vartheta_{1}(\pi z, \tau)=-\theta_{11}(z, \tau), \vartheta_{2}(\pi z, \tau)=$ $\theta_{10}(z, \tau), \vartheta_{3}(\pi z, \tau)=\theta_{00}(z, \tau), \vartheta_{4}(\pi z, \tau)=\theta_{01}(z, \tau)$.)

- We denote $\theta_{11}(z, \tau)$ by $[z]$ for simplicity.

- $[z]_{k}:=\prod_{j=0}^{k-1}[z+2 j \eta]=[z][z+2 \eta] \cdots[z+2(k-1) \eta]$ for $k=$ $1,2, \ldots,[z]_{0}=1$.

- The Pauli matrices are defined as usual:

$$
\sigma^{0}=\left(\begin{array}{ll}
1 & 0 \\
0 & 1
\end{array}\right), \quad \sigma^{1}=\left(\begin{array}{cc}
0 & 1 \\
1 & 0
\end{array}\right), \quad \sigma^{2}=\left(\begin{array}{cc}
0 & -i \\
i & 0
\end{array}\right), \quad \sigma^{3}=\left(\begin{array}{cc}
1 & 0 \\
0 & -1
\end{array}\right) .
$$

\section{Definition of the MOdel AND the $Q$-Operator}

In this section we define the generalisation of the eight vertex model, using the higher spin representations of the Sklyanin algebra. We mainly follow [28, with slightly different normalisation.

We fix a half integer $l \in \frac{1}{2} \mathbb{Z}_{>0}$ and consider the spin $l$ representation space of the Sklyanin algebra as the local state space $V_{i}(i=1, \ldots, N)$ : $V_{i} \cong \Theta_{00}^{4 l+}$. (See Appendix $\mathrm{A}$ for the Sklyanin algebra and its representations.) The total Hilbert space $\mathcal{H}$ is the tensor product of them:

$$
\mathcal{H}:=V_{N} \otimes \cdots \otimes V_{1},
$$

and the auxiliary space $V_{0}$ is a two-dimensional space: $V_{0} \cong \mathbb{C}^{2}$.

The transfer matrix $T(u)$ of the model acting on $\mathcal{H}$ is defined as follows:

$$
T(u):=\operatorname{tr}_{0} L_{N}(u) L_{N-1}(u) \cdots L_{1}(u),
$$

where the $L$-operator $L_{j}(u) \in \operatorname{End}_{\mathbb{C}}\left(\mathcal{H} \otimes V_{0}\right)$ is defined as

$$
L_{j}(u)=\sum_{a=0}^{3} W_{a}^{L}(u) \rho_{i}\left(S^{a}\right) \otimes \sigma^{a},
$$

by a representation of the Sklyanin algebra on $\mathcal{H}, \rho_{i}:=1 \otimes \cdots \otimes \rho^{l} \otimes$ $\cdots \otimes 1$, which acts non-trivially only on $V_{i}$. The functions $W_{a}^{L}(u)$ in (2.3) are defined by (A.3) in Appendix $\mathrm{A}$ and the matrices $\sigma^{a}$ are the Pauli matrices acting on $V_{0}$.

When the spin $l$ is $1 / 2$, the local quantum space $V_{i} \cong \Theta_{00}^{2+}$ can be identified with $\mathbb{C}^{2}$ by means of the basis $\left(\theta_{00}(2 z, 2 \tau)-\theta_{10}(2 z, 2 \tau), \theta_{00}(2 z, 2 \tau)+\right.$ $\left.\theta_{10}(2 z, 2 \tau)\right)$, and $\rho^{1 / 2}\left(S^{a}\right)$ are proportional to the Pauli matrices $\sigma^{a}$, 
(A.9). Therefore the transfer matrix $T(u)$ is essentially that of the eight vertex model. The $R L L$-relation (A.1) leads to the commutativity of the transfer matrix by the standard argument:

$$
T(u) T\left(u^{\prime}\right)=T\left(u^{\prime}\right) T(u) .
$$

The study of the model means the analysis of the spectrum of $T(u)$. The generalised algebraic Bethe Ansatz ([31]) has been successfully applied to this model in [27], [28] and [29]. However, when Baxter first solved the eight vertex model, he used the $Q$-operator instead.

The $Q$-operator is an invertible operator $Q(u): \mathcal{H} \rightarrow \mathcal{H}$, which is an entire function in a complex parameter $u$, satisfying the commutation relations:

$$
\begin{aligned}
& T(u) Q(u)=h_{-}(u) Q(u-2 \eta)+h_{+}(u) Q(u+2 \eta), \\
& Q(u) T(u)=h_{-}(u) Q(u-2 \eta)+h_{+}(u) Q(u+2 \eta), \\
& Q(u) Q(v)=Q(v) Q(u),
\end{aligned}
$$

where the functions $h_{ \pm}(u)$ are defined by

$$
h_{ \pm}(u):=(2[u \mp 2 l \eta])^{N} .
$$

Since $T$ is expressed in terms of $Q$, the relation (2.7) implies $[T(u), Q(v)]=$ 0 . We write down equivalent relations (2.5) and (2.6) separately, since they arise independently in the construction.

\section{Construction of the $Q$-operator}

In this section we construct the $Q$-operator for the higher spin eight vertex model defined in $\$ 2$. The main strategy is the same as that in Baxter's 1973 paper [2]:

(1) Find an auxiliary two-by-two matrix, a transformation by which makes the $(2,1)$-component of the $L$-operator degenerate. The tensor products of the null vectors of the twisted $(2,1)$-component are the column vectors of the $Q_{R^{-}}$operator satisfying the $T Q$ relation (2.5).

(2) Transposing $Q_{R}$ in an appropriate sense, we obtain the $Q_{L^{-}}$ operator which satisfies the $Q T$-relation (2.6).

(3) Show the commutativity $Q_{L}(u) Q_{R}(v)=Q_{L}(v) Q_{R}(u)$.

(4) The $Q$-operator is defined by $Q(u)=Q_{R}(u) Q_{R}\left(u_{0}\right)^{-1}=Q_{L}\left(u_{0}\right)^{-1} Q_{L}(u)$, where $u_{0}$ is a suitably fixed parameter.

3.1. Column vectors of $Q_{R}$. When we applied the generalised algebraic Bethe Ansatz of Takhtajan and Faddeev [31] in [27], 28] and [29], we used the gauge transformation matrix of the $L$-operator of the 
form 1

$$
M_{\lambda}(v):=\left(\begin{array}{rr}
-\theta_{00}((\lambda-v) / 2, \tau / 2) & -\theta_{00}((\lambda+v) / 2, \tau / 2) \\
\theta_{01}((\lambda-v) / 2, \tau / 2) & \theta_{01}((\lambda+v) / 2, \tau / 2)
\end{array}\right) .
$$

We denote the components of the twisted $L$-operator as follow: 2 .

$$
L_{\lambda, \lambda^{\prime}}(u ; v)=\left(\begin{array}{ll}
\alpha_{\lambda, \lambda^{\prime}}(u ; v) & \beta_{\lambda, \lambda^{\prime}}(u ; v) \\
\gamma_{\lambda, \lambda^{\prime}}(u ; v) & \delta_{\lambda, \lambda^{\prime}}(u ; v)
\end{array}\right):=M_{\lambda}(v)^{-1} L(u) M_{\lambda^{\prime}}(v),
$$

where $L(u)$ is the non-trivial part of $L_{j}(u)(2.3)$, i.e., the operator on $\Theta_{00}^{4 l+} \otimes \mathbb{C}^{2}$ defined by

$$
L(u)=\sum_{a=0}^{3} W_{a}^{L}(u) \rho^{l}\left(S^{a}\right) \otimes \sigma^{a} .
$$

It was shown in [27] (see also [28], [30]) that the operator $\gamma_{\lambda+4 l \eta, \lambda}(u ; v)$ degenerates and the following vector $\omega_{\lambda}(u ; v) \in \Theta_{00}^{4 l+}$ is a null vector:

$$
\omega_{\lambda}(u ; v):=\left[z+\frac{\lambda+u-v}{2}+(-l+1) \eta\right]_{2 l}\left[-z+\frac{\lambda+u-v}{2}+(-l+1) \eta\right]_{2 l},
$$

which is called the local pseudo vacuum in [30. This is a special case $(m=l)$ of the intertwining vectors in $\Theta_{00}^{4 l+}$ defined in [28] (see also [30], [23] and [24] $)^{3}$ :

$$
\begin{aligned}
\phi_{\lambda, \lambda^{\prime}}(u ; v) & :=\left[z+\frac{\lambda+u-v}{2}+(-l+1) \eta\right]_{l+m}\left[-z+\frac{\lambda+u-v}{2}+(-l+1) \eta\right]_{l+m} \times \\
& \times\left[z+\frac{\lambda^{\prime}+u-v}{2}+(-l+1) \eta\right]_{l-m}\left[-z+\frac{\lambda^{\prime}+u-v}{2}+(-l+1) \eta\right]_{l-m},
\end{aligned}
$$

where $\lambda^{\prime}=\lambda+4 m \eta(m \in-l, l+1, \ldots, l)$. The action of each component of $L_{\lambda, \lambda^{\prime}}(u ; v)$ on $\phi_{\lambda^{\prime}, \lambda}(u ; v)$ is known (cf. 28] (2.4) or [30] (1.19$22)$ ), among which we need the following three (cf. [28] (2.8-10) or [30] $(1.24-26))$ :

$$
\begin{aligned}
\alpha_{\lambda+4 l \eta, \lambda}(u ; v) \omega_{\lambda}(u ; v) & =2[u+2 l \eta] \omega_{\lambda-2 \eta}(u ; v), \\
\gamma_{\lambda+4 l \eta, \lambda}(u ; v) \omega_{\lambda}(u ; v) & =0 \\
\delta_{\lambda+4 l \eta, \lambda}(u ; v) \omega_{\lambda}(u ; v) & =\frac{2[u-2 l \eta][\lambda]}{[\lambda+4 l \eta]} \omega_{\lambda+2 \eta}(u ; v) .
\end{aligned}
$$

An important observation here is that the evenness of $\theta_{00}$ and $\theta_{01}$ implies the evenness of $M_{\lambda}(v)$ :

$$
M_{-\lambda}(-v)=M_{\lambda}(v) .
$$

\footnotetext{
${ }^{1}$ We use a different normalisation from those used in [27, 28]. The matrix $M_{\lambda}(u)$ here is the same as those used in [30] up to the shift of the parameter $\lambda \mapsto \lambda+1$ and the multiplication of a diagonal matrix from the right. 30 .

${ }^{2}$ The spectral parameters $u$ and $v$ here corresponds to $u-v$ and $u$ in (1.18) of

${ }^{3}$ As we changed the normalisation of $M_{\lambda}(v)$ in (3.1), the normalisation of intertwining vectors is different from that in 30 , by shift $\lambda \mapsto \lambda+1$.
} 
Therefore, along with the relations (3.6), we have

$$
\begin{aligned}
\alpha_{\lambda-4 l \eta, \lambda}(u ; v) \omega_{-\lambda}(u ;-v) & =2[u+2 l \eta] \omega_{-\lambda-2 \eta}(u ;-v), \\
\gamma_{\lambda-4 l \eta, \lambda}(u ; v) \omega_{-\lambda}(u ;-v) & =0, \\
\delta_{\lambda-4 l \eta, \lambda}(u ; v) \omega_{-\lambda}(u ;-v) & =\frac{2[u-2 l \eta][\lambda]}{[\lambda-4 l \eta]} \omega_{-\lambda+2 \eta}(u ;-v) .
\end{aligned}
$$

Moreover, because of the structure of the local pseudo vacuum vector (3.4), the shift of the auxiliary parameter $\lambda$ of $\omega_{\lambda}(u ; v)$ is equivalent to the shift of the spectral parameter $u$ :

$$
\omega_{\lambda \pm 2 \eta}(u ; v)=\omega_{\lambda}(u \pm 2 \eta ; v) .
$$

Hence, the relations (3.6) and (3.8) mean that, roughly speaking, the operator $\alpha$ (resp. $\delta$ ) shifts $u$ to $u-2 \eta($ resp. $u+2 \eta$ ).

Combining these facts, we construct the vector $\phi(u ; v, \lambda, \vec{\sigma})$, which will be a column vector of the operator $Q_{R}(u)$. Let us fix complex parameters $v$ and $\lambda$ and a sequence of $\pm 1, \vec{\sigma}=\left(\sigma_{N}, \sigma_{N-1}, \ldots, \sigma_{1}\right)$, which satisfies

$$
\sum_{k=1}^{N} \sigma_{k}=0
$$

(Since $N$ is even, there are $\left(\begin{array}{c}N \\ N / 2\end{array}\right)$ sequences satisfying this condition.) We define the sequence $\left\{\lambda_{j}\right\}_{j=1, \ldots, N+1}$ by

$$
\lambda_{1}=\lambda, \quad \lambda_{j+1}:=\lambda_{j}+4 \sigma_{j} l \eta=\lambda+4 \ln \sum_{k=1}^{j} \sigma_{k} .
$$

The condition (3.10) implies $\lambda_{N+1}=\lambda_{1}$. Because of the evenness (3.7) of $M_{\lambda}(v)$, we have

$$
\begin{aligned}
& (3.12) M_{\sigma_{j} \lambda_{j}+4 l \eta}\left(\sigma_{j} v\right)=M_{\lambda_{j}+4 \sigma_{j} l \eta}(v)=M_{\lambda_{j+1}}(v)=M_{\sigma_{j+1} \lambda_{j+1}}\left(\sigma_{j+1} v\right) . \\
& \left(\sigma_{N+1}:=\sigma_{1} .\right)
\end{aligned}
$$

Therefore, if we define a vector $g_{j}(u)=g_{j}(u ; v, \lambda, \vec{\sigma})$ in $V_{j}$ by

$$
g_{j}(u ; v, \lambda, \vec{\sigma}):=\omega_{\sigma_{j} \lambda_{j}}\left(u ; \sigma_{j} v\right)
$$

then the formulae (3.6) and (3.8) imply

$$
\begin{aligned}
\alpha_{\lambda_{j+1}, \lambda_{j}}(u ; v) g_{j}(u) & =\alpha_{\lambda_{j}+4 \sigma_{j} l \eta, \lambda_{j}}(u ; v) \omega_{\sigma_{j} \lambda_{j}}\left(u ; \sigma_{j} v\right) \\
& =2[u+2 l \eta] g_{j}(u-2 \eta), \\
\gamma_{\lambda_{j+1}, \lambda_{j}}(u ; v) g_{j}(u) & =\gamma_{\lambda_{j}+4 \sigma_{j} l \eta, \lambda_{j}}(u ; v) \omega_{\sigma_{j} \lambda}\left(u ; \sigma_{j} v\right) \\
& =0 \\
\delta_{\lambda_{j+1}, \lambda_{j}}(u ; v) g_{j}(u) & =\delta_{\lambda_{j}+4 l \eta, \lambda_{j}}(u ; v) \omega_{\sigma_{j} \lambda_{j}}\left(u ; \sigma_{j} v\right) \\
& =\frac{2[u-2 l \eta]\left[\lambda_{j}\right]}{\left[\lambda_{j+1}\right]} g_{j}(u+2 \eta) .
\end{aligned}
$$


Let us compute the action of the transfer matrix on the tensor product

$\phi(u ; v, \lambda, \vec{\sigma}):=g_{N}(u ; v, \lambda, \vec{\sigma}) \otimes g_{N-1}(u ; v, \lambda, \vec{\sigma}) \otimes \cdots \otimes g_{1}(u ; v, \lambda, \vec{\sigma}) \in \mathcal{H}$.

By insertion of $1=M_{\lambda_{j}}(v) M_{\lambda_{j}}(v)^{-1}$ between $L_{j}(u)$ and $L_{j-1}(u)$ in the definition (2.2) of the transfer matrix and by the cyclicity of the trace, we can rewrite the transfer matrix as

$$
T(u)=\operatorname{tr}_{0} \prod_{j=1, \ldots, N}^{\curvearrowleft}\left(\begin{array}{ll}
\alpha_{\lambda_{j+1}, \lambda_{j}}(u ; v) & \beta_{\lambda_{j+1}, \lambda_{j}}(u ; v) \\
\gamma_{\lambda_{j+1}, \lambda_{j}}(u ; v) & \delta_{\lambda_{j+1}, \lambda_{j}}(u ; v)
\end{array}\right)
$$

The formulae (3.14) reduces $T(u) \phi(u ; v, \lambda, \vec{\sigma})$ to a triangular form. Thus we obtain

$$
T(u) \phi(u ; v, \lambda, \vec{\sigma})=h_{-}(u) \phi(u-2 \eta ; v, \lambda, \vec{\sigma})+h_{+}(u) \phi(u+2 \eta ; v, \lambda, \vec{\sigma}) .
$$

Let $\left\{\phi_{k}(u):=\phi\left(u ; v_{k}, \lambda_{k}, \vec{\sigma}_{k}\right)\right\}_{k=1, \ldots, \operatorname{dim} \mathcal{H}}$ be a set of $\operatorname{dim} \mathcal{H}=(2 l+1)^{N}$ vectors with distinct values of parameters. We define a linear operator $Q_{R}(u): \mathbb{C}^{\operatorname{dim} \mathcal{H}} \rightarrow \mathcal{H}$ by

$$
Q_{R}(u): e_{k} \mapsto \phi_{k}(u),
$$

where $\left\{e_{k}\right\}_{k=1, \ldots, \operatorname{dim} \mathcal{H}}$ is a basis of $\mathbb{C}^{\operatorname{dim} \mathcal{H}}$. The $T Q$-relation (2.5) for $Q_{R}(u)$,

$$
T(u) Q_{R}(u)=h_{-}(u) Q_{R}(u-2 \eta)+h_{+}(u) Q_{R}(u+2 \eta),
$$

is a direct consequence of (3.17).

3.2. Hermitian conjugate and $Q_{L}$. As the next step, we construct an operator $Q_{L}(u)$ satisfying the " $Q T$ "-relation (2.6). For this purpose we study the Hermitian adjoint of $T(u)$. The space $\mathcal{H}$ has a natural Hermitian structure induced from the Sklyanin form (A.10) on $\Theta_{00}^{4 l+}$. We consider the adjoint operator with respect to this Hermitian structure.

Denote the four component of the $L$-operator (3.3) by $L_{\varepsilon \varepsilon^{\prime}}(u)\left(\varepsilon, \varepsilon^{\prime}=\right.$ $\pm)$ :

$$
L(u)=\left(\begin{array}{ll}
L_{--}(u) & L_{-+}(u) \\
L_{+-}(u) & L_{++}(u)
\end{array}\right) .
$$

By the definition (A.3) of the functions $W_{a}^{L}(u)$ and the self-adjointness (A.12) of $S^{a}$, it is easy to see that the adjoint operator of $L_{\varepsilon \varepsilon^{\prime}}$ is expressed by $L_{-\varepsilon,-\varepsilon^{\prime}}$ as follows:

$$
\begin{array}{lll}
\left(L_{--}(u)\right)^{*}=-L_{++}(-\bar{u}), & \left(L_{-+}(u)\right)^{*}=L_{+-}(-\bar{u}), \\
\left(L_{+-}(u)\right)^{*}=L_{-+}(-\bar{u}), & \left(L_{++}(u)\right)^{*}=-L_{--}(-\bar{u}) .
\end{array}
$$


Substituting the expression (3.20) into the definition (2.2) of the transfer matrix $T(u)$, we have

$$
\begin{aligned}
T(u) & =\sum_{\varepsilon_{N-1}, \ldots, \varepsilon_{1}= \pm} L_{N,-\varepsilon_{N-1}}(u) \cdots L_{j, \varepsilon_{j} \varepsilon_{j-1}} \cdots L_{1, \varepsilon_{1}-} \\
& +\sum_{\varepsilon_{N-1}, \ldots, \varepsilon_{1}= \pm} L_{N,+\varepsilon_{N-1}}(u) \cdots L_{j, \varepsilon_{j} \varepsilon_{j-1}}(u) \cdots L_{1, \varepsilon_{1}+}(u),
\end{aligned}
$$

where $L_{j, \varepsilon \varepsilon^{\prime}}(u)$ is the component of $L_{j}(u)$ defined by (2.3). Note that each term in the first sum corresponds bijectively to a term in the second sum by flipping all signs $\varepsilon_{j}$. On the other hand, there are as many $L_{-+}$as $L_{+-}$in each sum because of the boundary condition $\varepsilon_{N}=\varepsilon_{0}$ (= the \pm signs of the rightmost and the leftmost factors), which comes from the trace in (2.2). Therefore, in each summand in (3.22),

$$
\begin{aligned}
& \text { (number of } \left.L_{j,--}\right)+\left(\text { number of } L_{j,++}\right) \\
& =N-2\left(\text { number of } L_{j,+-}\right) \equiv N \equiv 0 \quad(\bmod 2) \text {. }
\end{aligned}
$$

The adjoint of $T(u)$ is obtained by substitution of (3.21) into (3.22):

$$
\begin{aligned}
(T(u))^{*} & =\sum_{\varepsilon_{N-1}, \ldots, \varepsilon_{1}=\mp} L_{N,+\varepsilon_{N-1}}(-\bar{u}) \cdots L_{j, \varepsilon_{j} \varepsilon_{j-1}}(-\bar{u}) \cdots L_{1, \varepsilon_{1}+}(-\bar{u}) \\
& +\sum_{\varepsilon_{N-1}, \ldots, \varepsilon_{1}=\mp} L_{N,-\varepsilon_{N-1}}(-\bar{u}) \cdots L_{j, \varepsilon_{j} \varepsilon_{j-1}}(-\bar{u}) \cdots L_{1, \varepsilon_{1}-}(-\bar{u}) \\
& =T(-\bar{u}) .
\end{aligned}
$$

The signs in (3.21) vanish in (3.24) because of (3.23).

Now let us take the adjoint of (3.19) with $-\bar{u}$ instead of $u$. We have

$$
\begin{aligned}
Q_{R}(-\bar{u})^{*} T(u) & =\overline{h_{-}(-\bar{u})} Q_{R}(-\bar{u}-2 \eta)^{*}+\overline{h_{+}(-\bar{u})} Q_{R}(-\bar{u}+2 \eta)^{*} \\
& =h_{+}(u) Q_{R}(-\bar{u}-2 \eta)^{*}+h_{-}(u) Q_{R}(-\bar{u}+2 \eta)^{*},
\end{aligned}
$$

because the theta function $[u]=\theta_{11}(u, \tau)$ is an odd real analytic function. (Here again we use the fact that $N$ is even.) Defining an operator $Q_{L}(u)$ by

$$
Q_{L}(u):=Q_{R}(-\bar{u})^{*}: \mathcal{H} \rightarrow \mathbb{C}^{\operatorname{dim} \mathcal{H}},
$$

we obtain the operator satisfying the relation (2.6):

$$
Q_{L}(u) T(u)=h_{-}(u) Q_{L}(u-2 \eta)+h_{+}(u) Q_{L}(u+2 \eta) .
$$

3.3. Commutation relation of $Q_{R}$ and $Q_{L}$. An important property of the operators $Q_{R}(u)$ and $Q_{L}(u)$ constructed above, (3.18) and (3.25), is the commutation relation:

$$
Q_{L}(u) Q_{R}\left(u^{\prime}\right)=Q_{L}\left(u^{\prime}\right) Q_{R}(u) .
$$


The $(i, j)$-element of $Q_{L}(u) Q_{R}\left(u^{\prime}\right)$ is

$$
\begin{aligned}
\left(e_{i}, Q_{L}(u) Q_{R}\left(u^{\prime}\right) e_{j}\right) & =\left(e_{i}, Q_{R}(-\bar{u})^{*} Q_{R}\left(u^{\prime}\right) e_{j}\right) \\
& =\left\langle Q_{R}(-\bar{u}) e_{i}, Q_{R}\left(u^{\prime}\right) e_{j}\right\rangle=\left\langle\phi_{i}(-\bar{u}), \phi_{j}\left(u^{\prime}\right)\right\rangle .
\end{aligned}
$$

where $($,$) is an Hermitian form on \mathbb{C}^{\operatorname{dim} \mathcal{H}}$ defined by $\left(e_{i}, e_{j}\right)=\delta_{i j}$. Hence, if the function $\Phi\left(u, u^{\prime}\right)$ of $\left(u, u^{\prime}\right)$ defined by

$$
\Phi\left(u, u^{\prime}\right):=\left\langle\phi(-\bar{u} ; v, \lambda, \vec{\sigma}), \phi\left(u^{\prime} ; v^{\prime}, \lambda^{\prime}, \vec{\sigma}^{\prime}\right)\right\rangle
$$

is symmetric in $u$ and $u^{\prime}$ for any choice of parameters $(v, \lambda, \vec{\sigma})$ and $\left(v^{\prime}, \lambda^{\prime}, \vec{\sigma}^{\prime}\right)$, the commutation relation (3.27) holds. By the definitions (3.15) and (3.13),$\Phi\left(u, u^{\prime}\right)$ is rewritten as

$$
\Phi\left(u, u^{\prime}\right)=\prod_{k=1}^{N}\left\langle\omega_{\sigma_{k} \lambda_{k}}\left(-\bar{u} ; \sigma_{k} v\right), \omega_{\sigma_{k}^{\prime} \lambda_{k}^{\prime}}\left(u^{\prime} ; \sigma_{k}^{\prime} v^{\prime}\right)\right\rangle .
$$

From the results of Rosengren [23] and [24] follows the factorisation of $\Phi\left(u, u^{\prime}\right)$.

Lemma 3.1. $\Phi\left(u, u^{\prime}\right)$ is factorised as

$$
\begin{aligned}
\Phi\left(u, u^{\prime}\right)= & C \prod_{k=1}^{N} F\left(\frac{\lambda_{k}^{\prime}-\bar{\lambda}_{k}}{2}+\frac{\sigma_{k}^{\prime} u^{\prime}+\sigma_{k} u}{2}+\left(\sigma_{k}^{\prime}-\sigma_{k}\right) l \eta+\frac{-v^{\prime}+\bar{v}}{2}\right) \times \\
& \times \prod_{k=1}^{N} G\left(\frac{\lambda_{k}^{\prime}+\bar{\lambda}_{k}}{2}+\frac{\sigma_{k}^{\prime} u^{\prime}-\sigma_{k} u}{2}+\left(\sigma_{k}^{\prime}-\sigma_{k}\right) l \eta+\frac{-v^{\prime}-\bar{v}}{2}\right) .
\end{aligned}
$$

Here the constant $C$ depends only on $\tau, \eta, l$ and $N$, while the functions $F(w)$ and $G(w)$ depend only on $\tau, \eta$ and $l$.

We prove this lemma in Appendix B.

This factorisation is exactly of the same type as (10.5.27) in [5] for the eight vertex model. The parameters $\lambda$ and $s_{j}$ in [5] correspond to $2 l \eta$ and $\lambda_{j} / 2$ here respectively. Baxter showed (p.219 (§10.5) of [5]) that a function with such a factorisation is symmetric in $u$ and $u^{\prime}$, whatever $F$ and $G$ are.

Thus the commutation relation (3.27) has been proved.

3.4. The $Q$-operator and its commutation relations. The rest of the construction of the $Q$-operator is the same as that in [2] and [5].

As in [2] and [5], we expect that varying parameters $(v, \lambda, \vec{\sigma})=$ $\left(v_{k}, \lambda_{k}, \vec{\sigma}_{k}\right)$ produces a set of sufficiently many vectors $\left\{\phi_{k}(u):=\phi\left(u ; v_{k}, \lambda_{k}, \vec{\sigma}_{k}\right)\right\}_{k=1, \ldots, \operatorname{dim} \mathcal{H}}$ which spans the space $\mathcal{H}$ for generic $u$, although we do not have a proof. Here we suppose this non-degeneracy and take a parameter $u=u_{0}$, for which $Q\left(u_{0}\right)$ has the inverse. We define the $Q$-operator by

$$
Q(u):=Q_{R}(u) Q_{R}\left(u_{0}\right)^{-1}: \mathcal{H} \stackrel{Q_{R}\left(u_{0}\right)^{-1}}{\longrightarrow} \mathbb{C}^{\operatorname{dim} \mathcal{H}} \stackrel{Q_{R}(u)}{\longrightarrow} \mathcal{H} .
$$


Then the commutation relation (3.27) implies

$$
\begin{gathered}
Q(u)=Q_{L}\left(u_{0}\right)^{-1} Q_{L}(u), \\
Q(u) Q\left(u^{\prime}\right)=Q_{L}\left(u_{0}\right)^{-1} Q_{L}(u) Q_{R}\left(u^{\prime}\right) Q_{R}\left(u_{0}\right)^{-1}=Q\left(u^{\prime}\right) Q(u) .
\end{gathered}
$$

Multiplying $Q_{R}\left(u_{0}\right)^{-1}$ from the right to (3.19), we have

$$
T(u) Q(u)=h_{-}(u) Q(u-2 \eta)+h_{+}(u) Q(u+2 \eta),
$$

while left multiplication of $Q_{L}\left(u_{0}\right)^{-1}$ to (3.26) leads to

$$
Q(u) T(u)=h_{-}(u) Q(u-2 \eta)+h_{+}(u) Q(u+2 \eta) .
$$

Thus we have all the commutation relations (2.5), (2.6), (2.7) and

$$
T(u) Q(u)=Q(u) T(u)
$$

from (2.5) and (2.6).

\section{The EIGEnVAlue of THE TRANSFer MATRIX}

As an application of the $Q$-operator, let us compute the eigenvalue of the transfer matrix $T(u)$ and prove the sum rule of the Bethe roots, which was proved in [28] under redundant conditions.

For the eight vertex model there are two involutive operators on $\mathcal{H}$ ( $R=$ (reversing the arrows) and $S=$ (assigning -1 to down arrows) in [5]) which commute with the transfer matrix. Baxter used them to break up $\mathcal{H}$ and deduced the Bethe Ansatz equation.

In our case we have also two involutions, $U_{1}^{\otimes N}$ and $U_{3}^{\otimes N}$, corresponding to $R$ and $S$. The definition of $U_{a}$, which was introduced by Sklyanin in [26], is given by (A.14) in Appendix A. It was shown in $\S 1.4$ of [28] that they commute with each other and with the transfer matrix $T(u)$.

$$
\left(U_{a}^{\otimes N}\right)^{2}=1, \quad\left[U_{a}^{\otimes N}, U_{b}^{\otimes N}\right]=\left[T(u), U_{a}^{\otimes N}\right]=0 .
$$

By the definition (A.14) of $U_{a}$, the operators $U_{1}$ and $U_{3}$ act on the local pseudo vacuum vector $\omega_{\lambda}(u ; v)$ defined by (3.4) as

$$
\begin{aligned}
& U_{1} \omega_{\lambda}(u ; v)=e^{-l \pi i} \omega_{\lambda}(u+1 ; v), \\
& U_{3} \omega_{\lambda}(u ; v)=e^{l \pi i(\tau-1)+2 l \pi i(\lambda+u-v+2 l \eta)} \omega_{\lambda}(u+\tau ; v) .
\end{aligned}
$$

Therefore operators $U_{a}^{\otimes N}$ act on the column vector $\phi(u ; v, \lambda, \vec{\sigma})$ of the $Q_{R^{-}}$operator defined by (3.15) as follows:

$$
\begin{aligned}
& U_{1}^{\otimes N} \phi(u ; v, \lambda, \vec{\sigma})=e^{-N l \pi i} \phi(u+1 ; v, \lambda, \vec{\sigma}), \\
& U_{3}^{\otimes N} \phi(u ; v, \lambda, \vec{\sigma})=e^{N l \pi i(\tau-1)+2 N l \pi i u} \phi(u+\tau ; v, \lambda, \vec{\sigma}) .
\end{aligned}
$$

Here we used a formula (the same as (10.5.40) in [5])

$$
\sum_{k=1}^{N} \sigma_{k} \lambda_{k}=-2 N l \eta
$$

which is a consequence of the condition (3.10) and the definition (3.11) of $\lambda_{j}$. Note that the coefficients of $\phi$ and the shifts of $u$ in (4.4) do not 
depend on the parameters $(v, \lambda, \vec{\sigma})$. Therefore the $Q_{R^{-}}$operator defined by (3.18) inherits those relations:

$$
\begin{aligned}
& U_{1}^{\otimes N} Q_{R}(u)=e^{-N l \pi i} Q_{R}(u+1), \\
& U_{3}^{\otimes N} Q_{R}(u)=e^{N l \pi i(\tau-1)+2 N l \pi i u} Q_{R}(u+\tau) .
\end{aligned}
$$

Because of the unitarity of the involution $U_{a}^{\otimes N}$ and the definition (3.25) of $Q_{L}(u)$, we have

$$
Q_{L}(u) U_{a}^{\otimes N}=\left(U_{a}^{\otimes N} Q_{R}(-\bar{u})\right)^{*} .
$$

When $a=1$, the product $U_{1}^{\otimes N} Q_{R}(-\bar{u})$ is equal to $e^{-N l \pi i} Q_{R}(-\bar{u}+1)$ by (4.5). Note that the property of the theta function $[z+1]=-[z]$ implies $\omega_{\lambda}(u+2 ; v)=\omega_{\lambda}(u ; v)$ and $Q_{R}(u+2)=Q_{R}(u)$. Hence

$$
U_{1}^{\otimes N} Q_{R}(\bar{u})=e^{-N l \pi i} Q_{R}(-\bar{u}-1)=e^{-N l \pi i} Q_{R}(-\overline{(u+1)}),
$$

which gives

$$
Q_{L}(u) U_{1}^{\otimes N}=e^{-N l \pi i} Q_{L}(u+1) .
$$

(Recall that $N l$ is an integer. Therefore $e^{-N l \pi i}$ is a real number \pm 1 .) Likewise we can prove

$$
Q_{L}(u) U_{3}^{\otimes N}=e^{N l \pi i(\tau-1)+2 N l \pi i u} Q_{L}(u+\tau) .
$$

Here we used $-\bar{u}+\tau=-\overline{(u+\tau)}$ and $e^{N l \pi i(\tau-1)} \in \mathbb{R}$, which are consequences of $\tau \in i \mathbb{R}$.

Multiplying $Q_{R}\left(u_{0}\right)^{-1}$ to (4.5) and (4.6) from the right and $Q_{L}\left(u_{0}\right)^{-1}$ to (4.7) and (4.8) from the left, we obtain

$$
\begin{aligned}
& U_{1}^{\otimes N} Q(u)=Q(u) U_{1}^{\otimes N}=e^{-N l \pi i} Q(u+1), \\
& U_{3}^{\otimes N} Q(u)=Q(u) U_{3}^{\otimes N}=e^{N l \pi i(\tau-1)+2 N l \pi i u} Q(u+\tau) .
\end{aligned}
$$

Having shown that the operators $T(u), Q(u)$ and $U_{a}^{\otimes N}$ commute with each other, we can consider the diagonalisation problem of $T(u)$ and $Q(u)$ on the joint eigenspaces of $U_{1}^{\otimes N}$ and $U_{3}^{\otimes N}$. Recall that $\left(U_{a}^{\otimes N}\right)^{2}=1$ (4.1). Hence the operators $U_{a}^{\otimes N}$ are diagonalisable and their eigenvalues are \pm 1 . Accordingly the space $\mathcal{H}$ is decomposed as

$$
\mathcal{H}=\bigoplus_{\nu_{1}, \nu_{3}=0,1} \mathcal{H}_{\nu_{1}, \nu_{3}},\left.\quad U_{a}^{\otimes N}\right|_{\mathcal{H}_{\nu_{1}, \nu_{3}}}=(-1)^{\nu_{a}} \operatorname{Id}_{\mathcal{H}_{\nu_{1}, \nu_{3}}}
$$

The rest is the same as in $\S 10.6$ of [5]. We suppose that $T(u)$ and $Q(u)$ are diagonalisable. Because of the commutativity, they are diagonalised simultaneously on each $\mathcal{H}_{\nu_{1}, \nu_{3}}$ by a matrix independent of $u$. Let us denote the eigenvalues of $T(u)$ and $Q(u)$ for one of the eigenvectors by $\Lambda(u)$ and $q(u)$, which are entire functions in $u$. The TQ-relation (2.5) gives the relation

$$
\Lambda(u) q(u)=h_{-}(u) q(u-2 \eta)+h_{+}(u) q(u+2 \eta) .
$$


The transformation rules (4.9) reduce to the scalar equations:

$$
\begin{aligned}
& (-1)^{\nu_{1}} q(u)=e^{-N l \pi i} q(u+1), \\
& (-1)^{\nu_{3}} q(u)=e^{N l \pi i(\tau-1)+2 N l \pi i u} q(u+\tau) .
\end{aligned}
$$

Applying the argument principle in the complex analysis, we can prove from (4.12) that the entire function $q(u)$ has $N l$ zeros in the rectangular with vertices $0,1,1+\tau$ and $\tau$. Let us denote these zeros by $u_{1}, \ldots, u_{N l}$. Note that $q(u)$ has zeros at $u_{j}+n+m \tau(n, m \in \mathbb{Z})$ as well because of the quasi-periodicity (4.12).

The theta function $[z]=\theta_{11}(z, \tau)$ has the quasi-periodicity:

$$
[z+1]=-[z], \quad[z+\tau]=-e^{-\pi i \tau-2 \pi i z}[z],
$$

and $[z]=0$ is equivalent to $z \in \mathbb{Z}+\tau \mathbb{Z}$. Hence the function

$$
f(u):=\frac{q(u)}{\prod_{j=1}^{N l}\left[u-u_{j}\right]}
$$

is entire, does not vanish and has the quasi-periodicity:

$$
f(u+1)=(-1)^{\nu_{1}} f(u), \quad f(u+\tau)=(-1)^{\nu_{3}} e^{-2 \pi i \sum_{j=1}^{N l} u_{j}} f(u)
$$

because of (4.12). By the standard argument in the complex analysis again, it follows from this periodicity that $f(u)$ is a constant multiple of $e^{K u}$, where the constant $K$ satisfies

$$
K=\nu_{1} \pi i+2 n_{1} \pi i, \quad K \tau=\nu_{3} \pi i-2 \pi i \sum_{j=1}^{N l} u_{j}+2 n_{3} \pi i
$$

for some integers $n_{1}$ and $n_{3}$. Putting the condition (4.15) back into the definition (4.14) of $f(u)$, we obtain the explicit form of $q(u)$

$$
q(u)=C e^{\nu_{1} \pi i u} \prod_{j=1}^{N l}\left[u-u_{j}\right]
$$

and the sum rules:

$$
\sum_{j=1}^{N l} u_{j} \equiv-\frac{\nu_{1} \tau}{2}+\frac{\nu_{3}}{2} \quad(\bmod \mathbb{Z}+\tau \mathbb{Z}) .
$$

Setting $u=u_{j}$ in (4.11), we have the equation

$$
h_{-}\left(u_{j}\right) q\left(u_{j}-2 \eta\right)+h_{+}\left(u_{j}\right) q\left(u_{j}+2 \eta\right)=0,
$$

or equivalently,

$$
\left(\frac{\left[u_{j}+2 l \eta\right]}{\left[u_{j}-2 l \eta\right]}\right)^{N}=e^{4 \nu_{1} \pi i \eta} \prod_{k=1, k \neq j}^{N l} \frac{\left[u_{j}-u_{k}+2 \eta\right]}{\left[u_{j}-u_{k}-2 \eta\right]},
$$

\footnotetext{
${ }^{4}$ The conjecture in 29 should be modified.
} 
which is nothing but the Bethe equation. The corresponding eigenvalue of the transfer matrix is

$$
\begin{aligned}
\Lambda(u) & =(2[u+2 l \eta])^{N} e^{-2 \nu_{1} \pi i \eta} \prod_{j=1}^{N l} \frac{\left[u-u_{j}-2 \eta\right]}{\left[u-u_{j}\right]} \\
& +(2[u-2 l \eta])^{N} e^{2 \nu_{1} \pi i \eta} \prod_{j=1}^{N l} \frac{\left[u-u_{j}+2 \eta\right]}{\left[u-u_{j}\right]},
\end{aligned}
$$

which was first obtained in [27] by the Bethe Ansatz.

\section{Concluding COMMENTS AND REMARKS}

We have constructed the $Q$-operator ((3.31) or $(3.32))$ satisfying the $T Q$-relation, (2.5) and (2.6), and commuting with itself, (2.7), for generalised eight vertex models defined by the higher spin representations of the Sklyanin algebra. Our explicit construction by means of the intertwining vectors makes it possible to prove the sum rule (4.17) of the Bethe roots, which we could not prove in [28] from the Bethe Ansatz itself. Thus the $Q$-operator can be useful to analyse the Bethe roots.

However there are several weak points in our construction. Let us make several comments on them with remarks on recent developments.

- Our construction as well as Baxter's original idea relies on the assumption that the operator $Q_{R}(u)$ is generically nondegenerate. (See (3.31).) This is very difficult to prove, although is plausible, as Baxter claimed on the basis of degenerate (six vertex) cases (p.220, [5]).

- The Bethe Ansatz for the higher spin generalisation of the eight vertex model in [27], [28] and [29] works when the total spin $\mathrm{Nl}$ is an integer. In particular, if $l$ is an integer, there is no constraint imposed on $N$. On the other hand, the construction of the $Q$-operator in this paper requires that $N$ is always even, especially because of the condition (3.10), $\sum \sigma_{k}=0$. In this respect our $Q$-operator method is weaker than the Bethe Ansatz.

- As is mentioned above, when the number of the lattice sites is odd, our construction does not work. In this case, we would need higher spin generalisation of Baxter's 1972 paper [1. There are many works (for example [14, [12], [15], [22]) in this direction for the eight vertex model.

- The $Q$-operators for the XXZ spin chain of higher spin were constructed by Roan in [21], following both ways of Baxter, [1] and [2]. The latter method is similar to ours, but since all the ingredients in the trigonometric case have explicit matrix description, there are no complication caused by the functional realisation of the representation spaces as in our case. (See also [19] for a related work.) 
- In addition to the difficulty of the non-degeneracy problem of $Q_{R}(u)$, there is another weakness of Baxter's classical method: the construction is essentially non-local because of $Q_{R}^{-1}$ in (3.31). Therefore it is not very useful for a further analysis of the model except its spectrum. Modern developments from different viewpoints 5 remove this weak point. In the context of the conformal field theory, Bazhanov, Lukyanov and Zamolodchikov ([7], [8]) suggested an essentially new approach, which gives $Q$-operators as traces of monodromy matrices in the trigonometric $\operatorname{spin}=1 / 2$ case. Mangazeev extended this construction to higher spin trigonometric cases in [17] (using the fusion procedure of the transfer matrices) and in [18] (as integral operators), based on the ideas of factorised $L$-operators [9]. Higher rank generalisation is found in [6]. These methods provide local construciton of $Q$-operators.

- The $Q$-operators for the elliptic models with infinite-dimensional state spaces were constructed by Zabrodin in [33] and by Chicherin, Derkachov, Karakhanyan and Kirschner in [10].

It is an interesting question, whether our $Q$-operator can be obtained by reduction from their $Q$-operators. In fact, recently Chicherin, Derkachov and Spiridonov [11] constructed a general elliptic $R$-operator acting in the tensor product of infinite dimensional representations of the Sklyanin algebra as the integral operator similar to [18] and restricted it to finite-dimensional representations. It would be remarkable, if such a restriction of elliptic $Q$-operators in [33] or [10] would be found, as they are constructed for any (i.e., even and odd) number of sites.

- Recently the $Q$-operator is studied from the viewpoint of representation theory of quantum algebras. See, for example, [13]. It is a challenging problem to understand elliptic $Q$-operators from the representation theory of elliptic quantum algebras.

Acknowledgements. The author dedicates this paper to Professor Evgeny Sklyanin on the occasion of his sixtieth birthday. It was Professor Sklyanin, who first led the author to the study of the elliptic quantum integrable models.

Special thanks are due to Hitoshi Konno, who suggested to use Rosengren's results in the proof of Lemma 3.1. The author also expresses his gratitude to Masahiko Ito, Saburo Kakei, Atsuo Kuniba, Masatoshi Noumi, Jun'ichi Shiraishi and Anton Zabrodin for discussions and encouragement.

The author is grateful to Rikkyo University, Tokyo, for its hospitality, where a part of this work was done.

\footnotetext{
${ }^{5}$ The author thanks the referee for informing the references in this remark and [1] in the next remark.
} 
The article was prepared within the framework of the Academic fund Program at the National Research University Higher School of Economics (HSE) in 2015-2016 (grant No.15-01-102) and supported within the framework of a subsidy granted to the HSE by the Government of the Russian Federation for the implementation of the Global Competitiveness Program.

\section{Appendix Appendix A SkLyAnin Algebra}

In this appendix we recall several facts on the Sklyanin algebra and its representations from [25] and [26].

The Sklyanin algebra is an associative algebra generated by four generators $S^{a}(a=0, \ldots, 3)$ subject to the following relations:

$$
L_{12}(v) L_{13}(u) R_{23}(u-v)=R_{23}(u-v) L_{13}(u) L_{12}(v) .
$$

Here the symbols are defined as follows:

- the $L$-operator $L(u)$ with a complex parameter $u$ is defined by

$$
L(u)=\sum_{a=0}^{3} W_{a}^{L}(u) S^{a} \otimes \sigma^{a}
$$

where 6

$$
\begin{aligned}
& W_{0}^{L}(u)=\frac{\theta_{11}(u, \tau)}{\theta_{11}(\eta, \tau)}, \quad W_{1}^{L}(u)=\frac{\theta_{10}(u, \tau)}{\theta_{10}(\eta, \tau)}, \\
& W_{2}^{L}(u)=\frac{\theta_{00}(u, \tau)}{\theta_{00}(\eta, \tau)}, \quad W_{3}^{L}(u)=\frac{\theta_{01}(u, \tau)}{\theta_{01}(\eta, \tau)} .
\end{aligned}
$$

- The matrix $R(u)$ is Baxter's R-matrix defined by

$$
R(u)=\sum_{a=0}^{3} W_{a}^{R}(u) \sigma^{a} \otimes \sigma^{a}, \quad W_{a}^{R}(u):=W_{a}^{L}(u+\eta) .
$$

- The indices designate the spaces on which operators act nontrivially. For example,

$L_{12}(u)=\sum_{a=0}^{3} W_{a}^{L}(u) S^{a} \otimes \sigma^{a} \otimes 1, \quad R_{23}(u)=\sum_{a=0}^{3} W_{a}^{R}(u) 1 \otimes \sigma^{a} \otimes \sigma^{a}$.

Although the relation (A.1) contains parameters $u$ and $v$, the commutation relations among $S^{a}(a=0, \ldots, 3)$ do not depend on them:

$$
\left[S^{\alpha}, S^{0}\right]_{-}=-i J_{\alpha, \beta}\left[S^{\beta}, S^{\gamma}\right]_{+}, \quad\left[S^{\alpha}, S^{\beta}\right]_{-}=i\left[S^{0}, S^{\gamma}\right]_{+},
$$

where $(\alpha, \beta, \gamma)$ stands for an arbitrary cyclic permutation of $(1,2,3)$ and $[A, B]_{ \pm}$are the (anti-)commutator $A B \pm B A$. The structure constants $J_{\alpha, \beta}=\left(\left(W_{\alpha}^{L}\right)^{2}-\left(W_{\beta}^{L}\right)^{2}\right) /\left(\left(W_{\gamma}^{L}\right)^{2}-\left(W_{0}^{L}\right)^{2}\right)$ depend on $\tau$ and $\eta$ but not on $u$.

\footnotetext{
${ }^{6}$ The functions $W_{a}^{L}(u)$ here are normalised differently from those in [30]: $W_{a}^{L(\text { here })}(u)=2 \theta_{11}(2 \eta, \tau) W_{a}^{L(\text { old })}$.
} 
Let $l$ be a positive half integer. The spin $l$ representation $\rho^{l}$ of the Sklyanin algebra is defined as follows: The representation space is a space of entire functions,

$$
\begin{aligned}
& \Theta_{00}^{4 l+}:=\{f(z) \mid \\
& \left.\quad f(z+1)=f(-z)=f(z), f(z+\tau)=\exp ^{-4 l \pi i(2 z+\tau)} f(z)\right\}
\end{aligned}
$$

which is of dimension $2 l+1$. The generator $S^{a}$ of the Sklyanin algebra acts as a difference operator on this space:

$$
\left(\rho^{l}\left(S^{a}\right) f\right)(z)=\frac{s_{a}(z-l \eta) f(z+\eta)-s_{a}(-z-l \eta) f(z-\eta)}{\theta_{11}(2 z, \tau)},
$$

where

$$
\begin{array}{ll}
s_{0}(z)=\theta_{11}(\eta, \tau) \theta_{11}(2 z, \tau), & s_{1}(z)=\theta_{10}(\eta, \tau) \theta_{10}(2 z, \tau), \\
s_{2}(z)=i \theta_{00}(\eta, \tau) \theta_{00}(2 z, \tau), & s_{3}(z)=\theta_{01}(\eta, \tau) \theta_{01}(2 z, \tau) .
\end{array}
$$

In the simplest case $l=1 / 2, \rho^{1 / 2}\left(S^{a}\right)$ are expressed by the Pauli matrices $\sigma^{a}$. We can identify $\Theta_{00}^{2+}$ and $\mathbb{C}^{2}$ by

$$
\begin{aligned}
& \theta_{00}(2 z, 2 \tau)-\theta_{10}(2 z, 2 \tau) \longleftrightarrow\left(\begin{array}{l}
1 \\
0
\end{array}\right), \\
& \theta_{00}(2 z, 2 \tau)+\theta_{10}(2 z, 2 \tau) \longleftrightarrow\left(\begin{array}{l}
0 \\
1
\end{array}\right) .
\end{aligned}
$$

Under this identification $S^{a}$ have matrix forms

$$
\rho^{1 / 2}\left(S^{a}\right)=\theta_{11}(2 \eta, \tau) \sigma^{a} .
$$

The representation space $\Theta_{00}^{4 l+}$ has a natural Hermitian structure defined by the following Sklyanin form:

$$
\langle f(z), g(z)\rangle:=\int_{0}^{1} d x \int_{0}^{\tau / i} d y \overline{f(z)} g(z) \mu(z, \bar{z})
$$

where $z=x+i y$ and the kernel function $\mu(z, w)$ is defined by

$$
\mu(z, w):=\frac{\theta_{11}(2 z, \tau) \theta_{11}(2 w, \tau)}{\prod_{j=0}^{2 l+1} \theta_{00}(z+w+(2 j-2 l-1) \eta, \tau) \theta_{00}(z-w+(2 j-2 l-1) \eta, \tau)} .
$$

The most important property of this sesquilinear positive definite scalar product is that the generators $S^{a}$ of the Sklyanin algebra become selfadjoint:
(A.12)
$\left(S^{a}\right)^{*}=S^{a}$, namely, $\left\langle f(z), S^{a} g(z)\right\rangle=\left\langle S^{a} f(z), g(z)\right\rangle$.

In [26] Sklyanin also defined involutive automorphisms:

$$
X_{a}:\left(S^{0}, S^{a}, S^{b}, S^{c}\right) \mapsto\left(S^{0}, S^{a},-S^{b},-S^{c}\right),
$$


for $a=1,2,3$, where $(a, b, c)$ is a cyclic permutation of $(1,2,3)$. The unitary operators $U_{a}$ defined by

$$
\begin{aligned}
& U_{1}: \Theta_{00}^{4 \ell+} \ni f(z) \mapsto\left(U_{1} f\right)(z)=e^{\pi i \ell} f\left(z+\frac{1}{2}\right), \\
& U_{3}: \Theta_{00}^{4 \ell+} \ni f(z) \mapsto\left(U_{3} f\right)(z)=e^{\pi i \ell} e^{\pi i \ell(4 z+\tau)} f\left(z+\frac{\tau}{2}\right),
\end{aligned}
$$

and $U_{2}=U_{3} U_{1}$, intertwine representations $\rho^{\ell} \circ X_{a}$ and $\rho^{\ell}: \rho^{\ell}\left(X_{a}\left(S^{b}\right)\right)=$ $U_{a}^{-1} \rho^{\ell}\left(S^{b}\right) U_{a}$. Operators $U_{a}$ satisfy the relations: $U_{a}^{2}=(-1)^{2 \ell}, U_{a} U_{b}=$ $(-1)^{2 \ell} U_{b} U_{a}=U_{c}$.

\section{Appendix Appendix B Proof of Lemma 3.1}

In this appendix we prove Lemma 3.1, using the results by Rosengren, 23] and [24]. (See also [16] for the detailed proof of formulae in the elliptic case.)

Rosengren [24] introduced the vectors of $\Theta_{00}^{2 N+}$,

$$
e_{k}^{N}(z ; a, b)=[z ; a]_{k}[z ; b]_{N-k},
$$

where $k=0,1, \ldots, N, a, b \in \mathbb{C}$ and

$$
[z ; a]:=[z+a][-z+a], \quad[z ; a]_{k}:=[z+a]_{k}[-z+a]_{k} .
$$

They form a basis of $\Theta_{00}^{2 N+}$ when the parameters $a$ and $b$ satisfy the genericity condition:

$$
\begin{gathered}
a-b+2 j \eta \notin \mathbb{Z}+\tau \mathbb{Z} \quad(j=1-N, 2-N, \ldots, N-1), \\
a+b+2 j \eta \notin \mathbb{Z}+\tau \mathbb{Z} \quad(j=0,1, \ldots, N-1) .
\end{gathered}
$$

Actually they are the same as the intertwining vectors (3.5) up to parametrisation:

$\phi_{\lambda, \lambda^{\prime}}(u ; v)=e_{l+m}^{2 l}\left(z ; \frac{\lambda+u-v}{2}+(-l+1) \eta, \frac{\lambda^{\prime}+u-v}{2}+(-l+1) \eta\right)$.

Rosengren studied the change of basis $\left\{e_{k}^{N}(z ; a, b)\right\}_{k=0, \ldots, N}$ to $\left\{e_{k}^{N}(z ; c, d)\right\}_{k=0, \ldots, N}$ in [24] and computed the Sklyanin form among them in [23]. We need the following facts from his results.

The coefficients $R_{k}^{l}(a, b, c, d ; N)$ (elliptic $6 j$-symbols) of the expansion ((5.4) in [24]; (3.4) in [16])

$$
e_{k}^{N}(z ; a, b)=\sum_{l=0}^{N} R_{k}^{l}(a, b, c, d ; N) e_{l}^{N}(z ; c, d)
$$

is expressed by the coefficients (elliptic binomial coefficients) of the expansion (the elliptic version of (3.9) in [24]; (3.2) in [16])

$$
[z ; a]_{k}=\sum_{n=0}^{k} C_{n}^{k}(a, b, c)[z ; b]_{n}[z ; c]_{k-n}
$$


as follows (the elliptic version of the equation before Theorem 3.3 in [24]; the last equation in $\S 4.3$ of [16]):

$$
R_{k}^{l}(a, b, c, d ; N)=\sum_{j=0}^{\min (k, l)} C_{j}^{k}(a, c, b+2(N-k) \eta) C_{l-j}^{N-j}(b, c+2 j \eta, d) .
$$

The explicit expression of $C_{n}^{k}(a, b, c)$ is also known (the elliptic version of (3.14) in [24]; (3.3) in [16]):

(B.7) $C_{n}^{k}(a, b, c)$

$$
=\frac{[2 \eta]_{k}}{[2 \eta]_{n}[2 \eta]_{n-k}} \frac{[a-c]_{n}[a+c+2(k-n) \eta]_{n}[a-b]_{k-n}[a+b+2 n \eta]_{k-n}}{[b-c+2(n-k) \eta]_{n}[c-b-2 n \eta]_{k-n}[b+c]_{k}} .
$$

What is necessary for us later is the extremal case $R_{N}^{N}(a, b, c, d ; N)$. Using (B.6) and (B.7), we have

$$
\begin{aligned}
R_{N}^{N}(a, b, c, d ; N) & =\sum_{j=0}^{N} C_{j}^{N}(a, c, b) C_{N-j}^{N-j}(b, c+2 j \eta, d) \\
& =\sum_{j=0}^{N} C_{j}^{N}(a, c, b) \frac{[b-d]_{N-j}[b+d]_{N-j}}{[c+2 j \eta-d]_{N-j}[c+2 j \eta+d]_{N-j}} \\
& =\frac{1}{[c-d]_{N}[c+d]_{N}} \times \\
& \times \sum_{j=0}^{N} C_{j}^{N}(a, c, b)[c-d]_{j}[c+d]_{j}[b-d]_{N-j}[b+d]_{N-j} .
\end{aligned}
$$

Comparing the last expression with (B.5), we obtain the formula:

$$
R_{N}^{N}(a, b, c, d ; N)=\frac{[d ; a]_{N}}{[d ; c]_{N}}
$$

(It is natural that $b$ does not appear in the right hand side, since $e_{N}^{N}(z ; a, b)$ does not depend on $b$. $)$

We also need the following orthogonality relation in $[23]^{7}$ (Theorem $3.4)$.

$$
\begin{aligned}
& \left\langle e_{l}^{N}\left(z ;-\bar{d}+(1-N) \eta+\frac{\tau+1}{2},-\bar{c}+(1-N) \eta-\frac{\tau+1}{2}\right), e_{k}^{N}(z ; c, d)\right\rangle \\
& =C_{N} e^{2 \pi i(-d l+c(N-l)-N(1+\tau) / 4)} \Gamma_{k}^{N}(c, d) \delta_{k, l}
\end{aligned}
$$

where

$$
C_{N}=\frac{-2 \eta e^{3 \pi i \tau / 4}}{[2(N+1) \eta] \prod_{j=1}^{\infty}\left(1-e^{2 j \pi i \tau}\right)^{3}}
$$

\footnotetext{
${ }^{7}\langle f, g\rangle$ in 23 is $\langle g, f\rangle$ in 26. It is linear in $f$ and conjugate linear in $g$. We follow the convention in 26 .
} 
is a constant depending only on $(\tau, \eta, N)$ and

(B.10)

$$
\begin{aligned}
\Gamma_{k}^{N}(c, d) & =e^{\pi i N(\tau-1) / 2} \frac{[c-d-2 N \eta]}{[c-d+2(2 k-N) \eta]} \times \\
& \times \frac{[2 \eta]_{k}[c-d+2 \eta]_{k}}{[-2 N \eta]_{k}[c-d-2 N \eta]_{k}}[c-d+2(1-N) \eta]_{N}[c+d]_{N} .
\end{aligned}
$$

In particular,

$$
\begin{aligned}
\Gamma_{N}^{N}(c, d) & =e^{\pi i N(\tau-1) / 2} \frac{[c-d-2 N \eta]}{[c-d+2 N \eta]} \times \\
& \times \frac{[2 \eta]_{N}[c-d+2 \eta]_{N}}{[-2 N \eta]_{N}[c-d-2 N \eta]_{N}}[c-d+2(1-N) \eta]_{N}[c+d]_{N} \\
& =e^{\pi i N(\tau+1) / 2}[c-d]_{N}[c+d]_{N} .
\end{aligned}
$$

Using these formulae, let us compute the Sklyanin form $\left\langle e_{N}^{N}(z ; \alpha, \beta), e_{N}^{N}(z ; \gamma, \delta)\right\rangle$. In the expansion of the form (B.4),

$$
e_{N}^{N}(z ; \gamma, \delta)=\sum_{n=0}^{N} R_{N}^{n}(\gamma, \delta, c, d ; N) e_{n}^{N}(z ; c, d),
$$

of the second factor, we choose the parameters $c$ and $d$, so that $\left\{e_{n}^{N}(z ; c, d)\right\}_{n=0, \ldots, N}$ is a dual basis (up to normalisation) to $\left\{e_{n}^{N}(z ; \alpha, \beta)\right\}_{n=0, \ldots, N}$. According to (B.9),

$$
\alpha=-\bar{d}+(1-N) \eta+\frac{\tau+1}{2}, \quad \beta=-\bar{c}+(1-N) \eta-\frac{\tau+1}{2},
$$

namely,

(B.13) $c=-\bar{\beta}+(1-N) \eta-\frac{-\tau+1}{2}, \quad d=-\bar{\alpha}+(1-N) \eta+\frac{-\tau+1}{2}$.

Applying $\left\langle e_{N}^{N}(z ; \alpha, \beta), \cdot\right\rangle$ to $(\underline{\mathrm{B} .12})$ and using the orthogonality relation (B.9), we have

$$
\begin{aligned}
& \left\langle e_{N}^{N}(z ; \alpha, \beta), e_{N}^{N}(z ; \gamma, \delta)\right\rangle \\
& =C_{N} e^{2 \pi i(-d N-N(1+\tau) / 4)} \Gamma_{N}^{N}(c, d) R_{N}^{N}(\gamma, \delta, c, d ; N) \\
& =C_{N} e^{2 \pi i(-d N-2 \pi i N(1+\tau) / 4)}\left(e^{\pi i N(\tau+1) / 2}[c-d]_{N}[c+d]_{N}\right)\left(\frac{[d ; \gamma]_{N}}{[d ; c]_{N}}\right) \\
& =C_{N} e^{-2 \pi i d N}[d ; \gamma]_{N} .
\end{aligned}
$$


Substituting (B.13),

(B.14)

$$
\begin{aligned}
& \left\langle e_{N}^{N}(z ; \alpha, \beta), e_{N}^{N}(z ; \gamma, \delta)\right\rangle \\
= & C_{N} e^{-2 \pi i N(-\bar{\alpha}+(1-N) \eta+(-\tau+1) / 2)} \\
\times & \prod_{j=0}^{N-1}\left[-\bar{\alpha}+(1-N) \eta+\frac{-\tau+1}{2}+\gamma+2 j \eta\right]\left[\bar{\alpha}-(1-N) \eta-\frac{-\tau+1}{2}+\gamma+2 j \eta\right] \\
= & C_{N} e^{\pi i N \tau / 2} \prod_{j=0}^{N-1} \theta_{00}(\gamma-\bar{\alpha}+(2 j-N+1) \eta, \tau) \theta_{00}(\gamma+\bar{\alpha}+(2 j+N-1) \eta, \tau) .
\end{aligned}
$$

In order to prove Lemma 3.1, we need to compute the Sklyanin form of two local pseudo vacuum vectors, $\omega_{\sigma \lambda}(-\bar{u} ; \sigma v)=\phi_{\sigma \lambda, \sigma \lambda+4 l \eta}(-\bar{u} ; \sigma v)$ and $\omega_{\sigma^{\prime} \lambda^{\prime}}\left(u^{\prime} ; \sigma^{\prime} v^{\prime}\right)=\phi_{\sigma^{\prime} \lambda^{\prime}, \sigma^{\prime} \lambda^{\prime}+4 l \eta}\left(u^{\prime} ; \sigma^{\prime} v^{\prime}\right)$. We identify them with the vectors $e_{2 l}^{2 l}(z ; a, b)$ by the formula (B.3), but not directly. For the later purpose, we should first modify the expression of $\omega_{\sigma \lambda}(u ; v)$,

$\omega_{\sigma \lambda}(u ; \sigma v)=\prod_{j=0}^{2 l-1}\left[z+\frac{\sigma \lambda+u-\sigma v}{2}+(2 j-l+1) \eta\right]\left[-z+\frac{\sigma \lambda+u-\sigma v}{2}+(2 j-l+1) \eta\right]$.

Since $[z]$ is an odd function and $\sigma= \pm 1$, it is rewritten as

$\omega_{\sigma \lambda}(u ; \sigma v)=\prod_{j=0}^{2 l-1}\left[z+\frac{\lambda+\sigma u-v}{2}+\sigma(2 j-l+1) \eta\right]\left[-z+\frac{\lambda+\sigma u-v}{2}+\sigma(2 j-l+1) \eta\right]$.

The set $\{\sigma(2 j-l+1) \eta \mid j=0, \ldots, 2 l-1\}$ is equal to $\{\sigma l \eta-(2 l-$ $1) \eta+2 j \eta \mid j=0, \ldots, 2 l-1\}$. Therefore we can identify $\omega_{\sigma \lambda}(u ; v)$ with $e_{2 l}^{2 l}$ as follows:

$$
\omega_{\sigma \lambda}(u ; \sigma v)=e_{2 l}^{2 l}\left(z ; \frac{\lambda+\sigma u-v}{2}+\sigma l \eta-(2 l-1) \eta, *\right),
$$

Here the second parameter in $e_{2 l}^{2 l}$ is irrelevant.

The final step of the proof is the computation of $\left\langle\omega_{\sigma \lambda}(-\bar{u} ; \sigma v), \omega_{\sigma^{\prime} \lambda^{\prime}}\left(u^{\prime} ; \sigma^{\prime} v^{\prime}\right)\right\rangle$. Taking (B.15) into account, we substitute $N=2 l$ and

$$
\begin{aligned}
& \alpha=\frac{\lambda-\sigma \bar{u}-v}{2}+\sigma l \eta-(2 l-1) \eta, \\
& \gamma=\frac{\lambda^{\prime}+\sigma^{\prime} u^{\prime}-v^{\prime}}{2}+\sigma^{\prime} l \eta-(2 l-1) \eta,
\end{aligned}
$$

into (B.14), which gives

$$
\begin{aligned}
& \left\langle\omega_{\sigma \lambda}(-\bar{u} ; \sigma v), \omega_{\sigma^{\prime} \lambda^{\prime}}\left(u^{\prime} ; \sigma^{\prime} v^{\prime}\right)\right\rangle \\
= & C_{2 l} e^{\pi i l \tau} \prod_{j=0}^{2 l-1} \theta_{00}\left(\frac{\lambda^{\prime}-\bar{\lambda}}{2}+\frac{\sigma^{\prime} u^{\prime}+\sigma u}{2}+\left(\sigma^{\prime}-\sigma\right) l \eta+\frac{-v^{\prime}+\bar{v}}{2}+(2 j-N+1) \eta, \tau\right) \\
\times & \prod_{j=0}^{2 l-1} \theta_{00}\left(\frac{\lambda^{\prime}+\bar{\lambda}}{2}+\frac{\sigma^{\prime} u^{\prime}-\sigma u}{2}+\left(\sigma^{\prime}+\sigma\right) l \eta-2(2 l-1) \eta+\frac{-v^{\prime}-\bar{v}}{2}+(2 j-N+1) \eta, \tau\right) .
\end{aligned}
$$


This is one of the factors in (3.29) and has the desired factorised structure as in (3.30).

\section{REFERENCES}

[1] Baxter, R. J.: Partition Function of the Eight-Vertex Lattice Model, Ann. Phys. 70 193-228 (1972)

[2] Baxter, R. J.: Eight-Vertex Model in Lattice Statistics and One-Dimensional Anisotropic Heisenberg Chain I, Ann. Phys. 76 1-24 (1973)

[3] Baxter, R. J.: Eight-Vertex Model in Lattice Statistics and One-Dimensional Anisotropic Heisenberg Chain II, Ann. Phys. 76 25-47 (1973)

[4] Baxter, R. J.: Eight-Vertex Model in Lattice Statistics and One-Dimensional Anisotropic Heisenberg Chain III, Ann. Phys. 76 48-71 (1973)

[5] Baxter, R. J.: Exactly solved models in statistical mechanics. Academic Press, Inc., London, (1982)

[6] Bazhanov, V. V., Frassek, R., Łukowski, T., Meneghelli, C. and Staudacher, M.: Baxter $Q$-operators and representations of Yangians, Nuclear Phys. B 850 148-174 (2011)

[7] Bazhanov, V. V., Lukyanov, S. L. and Zamolodchikov, A. B.: Integrable structure of conformal field theory II, $Q$-operator and DDV equation, Comm. Math. Phys. 190 247-278 (1997)

[8] Bazhanov, V. V., Lukyanov, S. L. and Zamolodchikov, A. B.: Integrable structure of conformal field theory III, The Yang-Baxter relation, Comm. Math. Phys. 200 297-324 (1999)

[9] Bazhanov, V. V. and Stroganov, Yu. G.: Chiral Potts model as a descendant of the six-vertex model, J. Statist. Phys. 59 799-817 (1990)

[10] Chicherin, D., Derkachov, S., Karakhanyan, D. and Kirschner, R.: Baxter operators with deformed symmetry, Nuclear Phys. B 868 652-683 (2013)

[11] Chicherin, D., Derkachov, S. E. and Spiridonov, V. P.: New elliptic solutions of the Yang-Baxter equation, arXiv:1412.3383,

[12] Fabricius, K.: A new Q-matrix in the eight-vertex model, J. Phys. A 40 40754086, (2007).

[13] Frenkel, E., Hernandez, D.: Baxter's relations and spectra of quantum integrable models, Duke Math. J. 164, 2407-2460 (2015)

[14] Fabricius, K. and McCoy, B. M.: New developments in the eight vertex model, J. Statist. Phys. 111, 323-337 (2003); ditto II, Chains of odd length, J. Stat. Phys. 120 37-70 (2005)

[15] Fabricius, K. and McCoy, B. M.: The TQ equation of the eight-vertex model for complex elliptic roots of unity, J. Phys. A 40, 14893-14926 (2007)

[16] Konno, H.: The vertex-face correspondence and the elliptic $6 j$-symbols, Lett. Math. Phys. 72 243-258 (2005)

[17] Mangazeev, V. V.: On the Yang-Baxter equation for the six-vertex model, Nuclear Phys. B 882 70-96 (2014)

[18] Mangazeev, V. V.: Q-operators in the six-vertex model, Nuclear Phys. B 886 166-184 (2014)

[19] Motegi, K.: On Baxter's Q operator of the higher spin XXZ chain at the Razumov-Stroganov point, J. Math. Phys. 54 063510, 13 pp. (2013)

[20] Mumford, D.: Tata Lectures on Theta I, Progress in Mathematics 28, Birkhuser, Boston, (1983)

[21] Roan, S.-S.: On Q-operators of XXZ Spin Chain of Higher Spin, arXiv: cond-mat/0702271.

[22] Roan, S.-S.: The $Q$-operator and functional relations of the eight-vertex model at root-of-unity $\eta=\frac{2 m K}{N}$ for odd $N, J$. Phys. A 40, 11019-11044 (2007) 
[23] Rosengren, H.: Sklyanin invariant integration, Int. Math. Res. Not. $603207-$ $3232(2004)$

[24] Rosengren, H.: An elementary approach to $6 \mathrm{j}$-symbols (classical, quantum, rational, trigonometric, and elliptic), Ramanujan J. 13 131-166 (2007)

[25] Sklyanin, E. K.: Some Algebraic Structures Connected with the Yang-Baxter Equation, Funkts. analiz i ego Prilozh. 16-4 27-34 (1982) (in Russian); Funct. Anal. Appl. 16 263-270 (1983) (English translation)

[26] Sklyanin, E. K.: Some Algebraic Structures Connected with the Yang-Baxter Equation. Representations of Quantum Algebras, Funkts. analiz i ego Prilozh. 17-4 34-48 (1983) (in Russian); Funct. Anal. Appl. 17 273-284 (1984) (English translation)

[27] Takebe, T.: Generalized Bethe Ansatz with the general spin representations of the Sklyanin algebra, J. Phys. A 25 1071-1083 (1992)

[28] Takebe, T.: Bethe ansatz for higher spin eight-vertex models, J. Phys. A 28 6675-6706 (1995); Corrigendum J. Phys. A 29 1563-1566 (1996)

[29] Takebe, T.: Bethe ansatz for higher-spin XYZ models - low-lying excitations, J. Phys. A 29 6961-6966 (1996)

[30] Takebe, T.: A system of difference equations with elliptic coefficients and Bethe vectors, Comm. Math. Phys. 183 161-181 (1997)

[31] Takhtajan, L. A. and Faddeev, L. D.: The quantum method of the inverse problem and the Heisenberg XYZ model, Uspekhi Mat. Nauk 34:5 13-63 (1979) (in Russian); Russian Math. Surveys 34:5 11-68 (1979) (English translation)

[32] Whittaker, E. T. and Watson, G. N.: A course of modern analysis, An introduction to the general theory of infinite processes and of analytic functions; with an account of the principal transcendental functions, the fourth edition, Cambridge University Press, New York (1927)

[33] Zabrodin, A.: Commuting difference operators with elliptic coefficients from Baxter's vacuum vectors, J. Phys. A 33 3825-3850 (2000)

Faculty of Mathematics, National Research University Higher School of Economics, Vavilova Street 7, Moscow, 117312, Russia.

E-mail address: ttakebe@hse.ru 